\title{
Combining multiple influence strategies to increase consumer compliance
}

\author{
Maurits Kaptein*
}

Department of Statistics and Research Methods,

University of Tilburg,

Room P 1106, P.O. Box 90153,

5000 LE Tilburg, The Netherlands

E-mail: maurits@mauritskaptein.com

*Corresponding author

\section{Steven Duplinsky}

\author{
RPX Corporation, \\ 767G Portola Street, \\ San Francisco CA, 94129, USA \\ E-mail: steven@duplinsky.com
}

\begin{abstract}
In this paper, we investigate the effects and implications of utilising multiple social influence strategies simultaneously to endorse a single product or call to action. In three, studies we show that combinations of social influence strategies do not increase compliance - this is contrary to commonly held beliefs and practice. Studies 1 and 2 show that combining implementations of both the consensus and authority strategies to promote a single behaviour does not lead to an increase in the effectiveness of a persuasive attempt. In Study 3, we test these findings in an online advertising campaign and again show that a single influence strategy is more effective than the combined usage of multiple influence strategies. The paper outlines the importance of appropriately choosing and implementing social influence strategies to prevent unintended interactions between the strategies that lead to a suboptimal performance.
\end{abstract}

Keywords: persuasive technology; influence strategies; adaptive systems; online commerce; recommender systems.

Reference to this paper should be made as follows: Kaptein, M. and Duplinsky, S. (2013) 'Combining multiple influence strategies to increase consumer compliance', Int. J. Internet Marketing and Advertising, Vol. 8, No. 1, pp.32-53.

Biographical notes: Maurits Kaptein is an Assistant Professor at the Department of Statistics and Research Methods at the Tilburg School of Social and Behavioural Sciences. He is also a co-founder and the Chief Scientist at Science Rockstars. In his work, he examines people's responses to persuasion, with a focus on heterogeneity in such responses. Over the last few years, he has worked on the theoretical implications and the practical application of persuasion profiles. His work has been published both in Marketing (e.g., the Journal of Interactive Marketing) communications (e.g., the Journal of Computer Mediated Communication), and computer science (e.g., IEEE Transactions on Interactive Systems). 
Steven Duplinsky is currently the Product Manager at RPX Corporation in San Francisco CA, USA. He graduated with Honours from Stanford University in the Department of Communications where he focused on collaborative systems design and their influence on innovation and productivity in the modern workforce as well as research on means-based adaptive persuasive systems with Dr. Kaptein. Since graduating, he has been working in industry and was a Guest Instructor at Stanford. His research interests include collaborative systems, adaptive persuasive systems, and lower elaboration persuasive techniques.

\section{Introduction}

Peripheral strategies pervade persuasive appeals used by online retailers to advertise their products and services in order to promote efficacious attitudes and behaviours in their consumers. Vendors do not merely present functionally descriptive information about the products offered but provide additional qualitative appeals, such as 'bestseller', 'editor's pick', or 'limited time offer'. The use of high level persuasive appeals such as these has long been practiced by brick and mortar retailers and has seen a recent boom in digital commerce due to the easily adaptable nature of digital storefronts. In this paper, we argue that these messages are effective, on average, because they are implementations of more broadly defined social influence strategies, or what are considered influence tactics in the management literature. Through three empirical studies, we examine whether combining implementations of multiple of these influence strategies to support one single product proposition more favourably affects consumer behaviour than the implementation of a single influence strategy.

\subsection{Social influence strategies}

Social Influence Strategies are persuasive appeals that speak to an individual's social and conscious mind. Numerous taxonomies of different types of social influence strategies exist: Cialdini (2001) identifies six social influence strategies, while Rhoads (2007) identifies over a hundred different tactics. The differences in quantity arise from a choice to either group a number of implementations of persuasive appeals into one single influence strategy - referably one in which the psychological mechanisms for the effectiveness of an implementation are similar (Cialdini, 2001, 2004, 2005) - as compared to separately naming and identifying each influence attempt (see e.g., Kaptein and Eckles, 2010; Kaptein et al., 2011b). In this paper, we study the usage of three of the most pervasive social influence strategies that are represented in a similar respect in most of the scientific taxonomies and prominently used in online sales and marketing: consensus, authority, and scarcity. 


\subsubsection{Consensus}

Innate social and risk mitigation tendencies of humanity make individuals more comfortable forming an opinion or taking an action previously completed by numerous other individuals. This principle, that of consensus, is implemented frequently throughout online commerce settings. Consensus appeals often take the form of a 'bestseller' notification or positive 'star' evaluations by other customers. Highlighting observations of others who are engaging in the same belief or behaviour convinces people to believe and behave in similar ways (Ajzen and Fishbein, 1980; Cialdini, 2004; Goldstein et al., 2008; Zhu and Zhang, 2010). Ascribed to the effectiveness of the consensus strategy is the notion of conformity, as well as the postulation that the appeal serves as an informational influence by appearing as 'social proof' (Hardin and Higgins, 1996; Cialdini, 2001). Modern social media has revolutionised the applicability and relevance of consensus appeals; allowing for specific, accurate, and targeted messages. Most commonly, consensus appeals are used to build trust in a product or vendor by collating individual reviews into an overall product or site rating. However, much simpler appeals such as "Over 1 Million Customers!" are prevalent in more generalised advertising.

Due to the prevalence of consensus appeals currently in use in advertising and the fitting juxtaposition of consensus strategies with authority strategies, we use consensus appeals in all three studies described in this paper. For Studies 1 and 2, we had the benefit of knowing our target demographic (students) and were able to tailor the consensus description accordingly. In Study 3, we used a broader, simplistic consensus appeal and replicated our results in a less knowledgeable market context. In all cases, we used careful pre-testing to ensure the consensus appeal was valid in the context which we described.

\subsubsection{Authority}

Endorsements from authority figures frame messages in the context of a societally respectable figure or leader. These authority appeals are targeted to lead an individual's framing of the targeted product or desired behaviour to align with the authority. Previous research supports these practices and has shown that typically when an authority figure tells people to do something, they will comply (Milgram, 1974; Blass, 1991). As an evolutionary form of social influence (Kelman and Hamilton, 1989; Martin and Hewstone, 2003), the authority appeal speaks to one's basic understanding of the functioning of every social community through various levels of authority and obedience (Modigliani and Rochat, 1995; Cialdini, 2001). The simplicity and general applicability of authority appeals make them attractive to change or influence consumer behaviour.

We used authority arguments in all three studies to follow. In Studies 1 and 2, we carefully balanced the strength of the authority argument with respect to the consensus arguments in order to isolate our theory of dis-congruence in strength of persuasive strategies implemented simultaneously.

\subsubsection{Scarcity}

Appealing to another innate psychological trait - need for uniqueness (Fromkin, 1970; Snyder and Fromkin, 1980) - many vendors implement the scarcity strategy to make 
products appear exclusive, rare, or one-of-a-kind. These attributes of the products are then translated to feelings by the consumer who, according to commodity theory (Brock, 1968), desires scarce products more because the possession of such products produces feelings of personal distinctiveness or uniqueness. Phrases such as 'limited release' and 'while supplies last' (Lynn, 1991) are used to favourably affect consumer attitudes and increase the chance of purchase (West, 1975; Inman et al., 1997; Eisend, 2008; Lynn, 1989). This assumed scarcity increases the perceived value of products and opportunities (Cialdini, 2001).

In the context of online advertising, scarcity plays an important role due to the relative lack of need of contextual awareness and low elaboration. Study 3 implements scarcity arguments for the first time. We used it to provide additional depth to findings of Studies 1 and 2 as we built on the previous results with Study 3.

\subsection{Influence strategies for a low elaboration, high frequency society}

To describe the process by which social influence strategies are effective in changing consumers' attitudes or behaviours, researchers frequently turn to dual processing models of persuasive appeals. The most popular of such models is the elaboration likelihood model (ELM) (Petty and Cacioppo, 1986; Petty and Wegener, 1999; Booth-Butterfield and Welbourne, 2002) which defines two main approaches to persuading an individual to perform a desired action: the high elaboration, central route and the low elaboration, peripheral route.

The former, central route, invokes high elaboration by using logic and reason to convince the target individual to perform a desired action. Benefits to high elaboration are increased mental commitment by the target and an opportunity to develop a more thorough and lasting persuasive impact. However, high elaboration exposes a weak argument to greater potential for rejection and, due to requiring engagement of the target for longer periods of time, is feasibly more difficult to implement. The second approach, the peripheral route, relies on low elaboration through effective and concise persuasive strategies that invoke predefined feelings or attitudes in a target rather than elaborate reasoning or logic. Peripheral approaches are beneficial due to the ease with which they can be implemented and relatively low risk of rejection by the target. However, it is more difficult to establish a strong commitment from the target through peripheral processing.

With advertisements increasing in volume and frequency, opportunities for elaboration have decreased bringing more prominence to peripheral, low elaboration strategies. Modern consumers are barraged by pleas for consumption in every part of their lives; from banner ads on Smartphone applications to targeted ads on their favourite blogs. The average person can no longer escape the persistent ecosystem of advertising appeals as these appeals become worked increasingly deeper into every part of daily life.

Implementations of social influence strategies are theorised to function mainly through a low elaboration, peripheral route since, as described above, most common strategies rely on innate psychological tendencies rather than guided logic and reason. Our findings will also show that incongruent peripheral persuasive strategies run the risk of increasing target elaboration and decreasing compliance. Thus, suggesting a dependency among appeals that can result in a substantive change of elaboration model for a given appeal based on simultaneously co-present like-process appeals 
(i.e., combining multiple low elaboration appeals) could run the risk of creating unintended high elaboration.

\subsection{Using multiple influence strategies}

Since the social psychology literature identifies several influence strategies, such as consensus, authority, and scarcity, which are the focus of this paper, it is unclear to practitioners whether it is beneficial to use implementations of as many social influence strategies as possible to endorse a single product, or rather to pick the most effective one (Kaptein et al., 2011a). Thus, if a product is both recommended by an expert and the product is a bestseller (consensus) should a vendor combine these messages when presenting a product to a consumer or is the vendor better off selecting an influence strategy? Within the marketing literature two teams of researchers have tried to answer this question.

Falbe and Yukl (2008) queried the targets of influence attempts about their experiences when being influenced. Next to finding that some strategies are more effective than others in the context of motivating employees to perform certain activities, they also report that combining multiple strategies leads to increased compliance. For a number of influence strategies that they define, the effects are greater when they are combined or presented in sequence. However, the authors do mention in their discussion that most of their observations involved initial influence attempts which may bias the results of the use of single strategies: this is more likely to be unsuccessful given the setup of their study.

Barry and Shapiro (1992) also consider the usage of multiple influence strategies in their work on compliance gaining in dyadic relationships. Their results are in sharp contrast with those presented by Falbe and Yukl (2008): The combination of multiple strategies repeatedly leads to lowered compliance. Given the controlled nature of the experiment that is presented by Barry and Shapiro (1992) these results might pose higher internal validity then those presented by Falbe and Yukl (2008). However, the results presented by the latter seem more externally valid given the retrospective examination of real influence attempts. In this paper, we present an empirical test of the effects of the usage of multiple strategies both in a controlled experiment (Studies 1 and 2) as well as in a field (Study 3).

\subsection{Overview}

Given that multiple psychological processes are simultaneously in play in a single individual, and that - depending on the individual - these processes may or may not all support the product proposition or compliance request, it is important to examine the effects and interplay of simultaneous implementation of multiple social influence strategies. We hypothesise - contrary to current marketing practice but in-line with the findings presented by Barry and Shapiro (1992) - that combining multiple influence strategies to support a single appeal is not necessarily beneficial. The subsequent studies were designed to explore this hypothesis and apply the findings in both a scientific and industrial context.

In Study 1, we experimentally examine this hypothesis by developing implementation of the consensus and authority strategies. We bias the latter to be 
Combining multiple influence strategies to increase consumer compliance 37

preferred in the context of the given request. By presenting participants either with only one of the two influence strategies or with implementations of both strategies simultaneously, we show that compliance is significantly lower when both strategies are present. Given that we consciously biased one of the strategies in Study 1 to be able to demonstrate a possible detrimental effect of combining implementations of multiple strategies for a single appeal, we extended our finding by examining a similar situation in which the implementations of each of the strategies are equally preferred. Study 2 shows that usage of additional strategies - even if compliance to each of them is positive when used in isolation - does not increase compliance to a request.

Study 3 tests these same principles in a externally valid market setting. We test the effects of different influence strategies and their possible combinations on the click-through behaviour of online consumers. This study clearly shows the potential negative effects of combining of multiple influence strategies.

\section{Study 1: combining multiple strategies}

In Study 1, we test, using an experimental compliance task, whether combining implementations of multiple influence strategies toward the same appeal leads to increased compliance over the implementation of only one single influence strategy. Specifically, we test whether using both the authority and the consensus strategy increases compliance compared to using only a single strategy. To further determine the relationships of multiple sources and their effects on compliance, we included two groups of multiple strategy appeals for which, in one group, the expert and consensus had congruent (i.e., agreeing) recommendations and one group for which the recommendations were dis-congruent (i.e., disagreeing).

\subsection{Method}

\subsubsection{Participants}

Forty-four undergraduate students volunteered to participate by accepting an e-mail invitation and following a link to the study website. Participants were recruited from the communication and computer science departments. The e-mail invitation was sent to a total of 136 possible participants, resulting in a $32.4 \%$ response rate. The final sample consisted of $25(56.8 \%)$ females. The average age of the sample was $23.8(\mathrm{SD}=7.6)$.

\subsubsection{Procedure}

Once participants followed the link on the e-mail invitation to the online study, they were presented with an introduction page that included a consent waiver and a request to set aside approximately 30 minutes of their time to complete the study. The first part of the study consisted of an 'arctic survival item-ranking task'. Participants were asked to rank 12 items in order of importance after being given the following scenario:

You have just survived the crash of a small plane.

Both the pilot and co-pilot were killed in the crash.

It is mid-January, and you are in Northern Canada. 
The daily temperature is 25 below zero, and the night time temperature is 40 below zero. There is snow on the ground, and the countryside is wooded with several creeks criss-crossing the area. The nearest town is 20 miles away. You are dressed in city clothes appropriate for a business meeting. You manage to salvage twelve items that you can use to try to survive.

Participants then ranked items from 1 (most important to survival) to 12 (least important to survival):

1 a $20^{\prime} \times 20^{\prime}$ piece of heavy-duty canvas

2 a cigarette lighter

3 a compass

4 dehydrated milk (eight pounds)

5 duct tape (25' roll)

6 an extra shirt and pair of pants for each survivor

7 a hand ax

8 iodine water purification tablets (50 tablets)

9 a loaded .45 -caliber pistol

10 a loud signal whistle

11 one box of matches

12 a sectional air map made of plastic.

Once participants were finished ranking these items, a period of six seconds was spent (ostensibly) analysing their ranking before participants were told "...some of your rankings were correct, but some could use improvement. You will now get the chance to revise your answers".

\subsubsection{Manipulations}

In this experiment, we used four conditions on two dimensions:

a the number of strategies used (one or multiple)

b whether, in the multiple strategy condition, the implementations were congruent (yes or no).

In the single strategy condition, participants received 'advice' on how to change their rankings either from an expert or based on the consensus of a group of similar others. Participants were told: "You will have the chance to revise your answers based on advice from..." and then were shown a picture and a brief textual description of their advice source. Despite the different source labels, all participants were exposed to the same advice. Participants in the single strategy condition were randomly assigned to either the authority or consensus condition. The authority strategy was implemented as follows: Participants were told that they would receive advice originating from a 
'survival expert'. This was supported with the notion that: "You will get tips on how to better rank your items based on the knowledge of an arctic expert". The consensus strategy was implemented by stating that participants would receive advice from 'other students' and was further elaborated on by stating: "You will get tips on how to improve your ranking of items based on the consensus of other students who have generally done well on these types of problems".

To confirm the authority strategy, as we described in this study, was clearly preferential under these circumstances over the consensus strategy, we sent a short pretest via e-mail to 145 student outside of the recruitment pool that would be used for the study. The pretest participants were introduced to the disaster scenario exactly as would be used in the study. They were then presented with the description of the two sources exactly as they would appear in the study and asked to choose from which 'source' (authority vs. consensus) they would prefer to receive survival advice. Of the 145 pre-test participants, 144 self-selected the authority advice as the most preferential source of advice. These results provided conclusive support that the experimental design correctly biased the desirability of the sources as intended.

Congruence of sources was the nested condition within the multiple strategy dimension. In the congruent condition, the advice of both sources agreed. The message read: "The group of successful students agreed with the expert" for five out of the six suggestions. To increase realism, the sixth suggestion stated that the sources disagreed with each other. These two numbers were reversed in the incongruent condition so that the two sources disagreed on five out of six suggestions and agreed on one.

These are the four experimental groups in the experiment:

1 Single strategy-authority. Advice from the authority source only. $N=10$.

2 Single strategy-consensus. Advice from the consensus source only. $N=12$.

3 Multiple strategy-congruent. Agreeing advice from the authority source and consensus source. $N=10$.

4 Multiple strategy-incongruent. Disagreeing advice from the authority source and consensus source. $N=12$.

\subsubsection{Measures}

Compliance was measured by the degree to which a participant adhered to the advice from the source(s) for each of the six suggestions. All participants received the same suggestions for item ranking improvement in the same order. The compliance score is the sum of the number of ranks changed between initial rank and suggested ranks for the items for which a re-rank was suggested. Hence, if the item that was initially ranked at 1 was moved to position 5 (suggested position was 7) in the final rankings, participants received a score of $(|7-1|-|7-5|)=4$, (the maximal suggested change minus the actual distance between the suggested rank and the final rank) for that item. The maximum compliance score was $(6+2+7+6+7+2=) 28$. Negative scores could be obtained when items were moved in greater rank-distance to the suggestions than the initial rank, however, this did not occur in the study. 
Besides the actual compliance score, we measured participant's confidence in their final ranking. Participants' confidence in the final ranking was measured using the following two items:

1 How confident are you in your final ranking?

2 How satisfied are you with your final ranking?

Only the endpoints of the ten-point scales were labelled: 'Not at all confident' to 'Very confident' for the first item and 'Not at all satisfied' to 'Very satisfied' for the second item.

\subsection{Results}

For each of the dependent variables, we first looked at a main-effect of the number of strategies. Next, separate analyses were performed to test the effects of the specific strategy that was used, the number of strategies used, and the congruency of the messages under the multiple strategy condition.

\subsubsection{Compliance}

For the actual compliance to the advice given in the four experimental conditions, we found no significant main-effect of the number of sources: The average compliance score for the single source conditions, $\bar{X}=15.8, S E=1.96$, was similar to that of the multiple strategy condition, $\bar{X}=17.2, S E=1.62 t(42)=0.55, p=.582$. Within the single strategy condition a strong effect - as expected based on the pre-test for Study 1 - of the actual strategy that was used was found: Participants in the authority condition, $\bar{X}=23.6, S E=1.87$, complied much more to the advice than participants in the consensus condition, $\bar{X}=9.3, S E=1.61, t(20)=5.80, p<.001$. Within the multiple strategy condition both the incongruent group, $\bar{X}=17.6, S E=2.34$, and the congruent group, $\bar{X}=16.8, S E=2.33$ had similar mean compliance scores, $t(20)=0.235, p=.816$.

Figure 1 shows the means and standard errors for each of the experimental groups. When conducting a one-way four level ANOVA on this data, there is a significant main-effect of condition, $F(3,40)=8.097, p<.001$. Bonferroni corrected pairwise comparisons show that the single strategy consensus condition scores significantly lower than all other conditions, while the single strategy authority condition scores significantly higher. Table 1 shows the mean differences between each of the four conditions, their standard errors, and the $p$-value for each possible pairwise comparison. The results indicate that when choosing the optimal single strategy for a specific context, adding other strategies can have a detrimental effect on compliance. It further shows that when the effect of one of the sources is clearly preferable, the effects of incongruence are insignificant. 
Combining multiple influence strategies to increase consumer compliance 41

Figure 1 The effects of the use of single or multiple strategies, either authority or consensus and congruent or incongruent on compliance

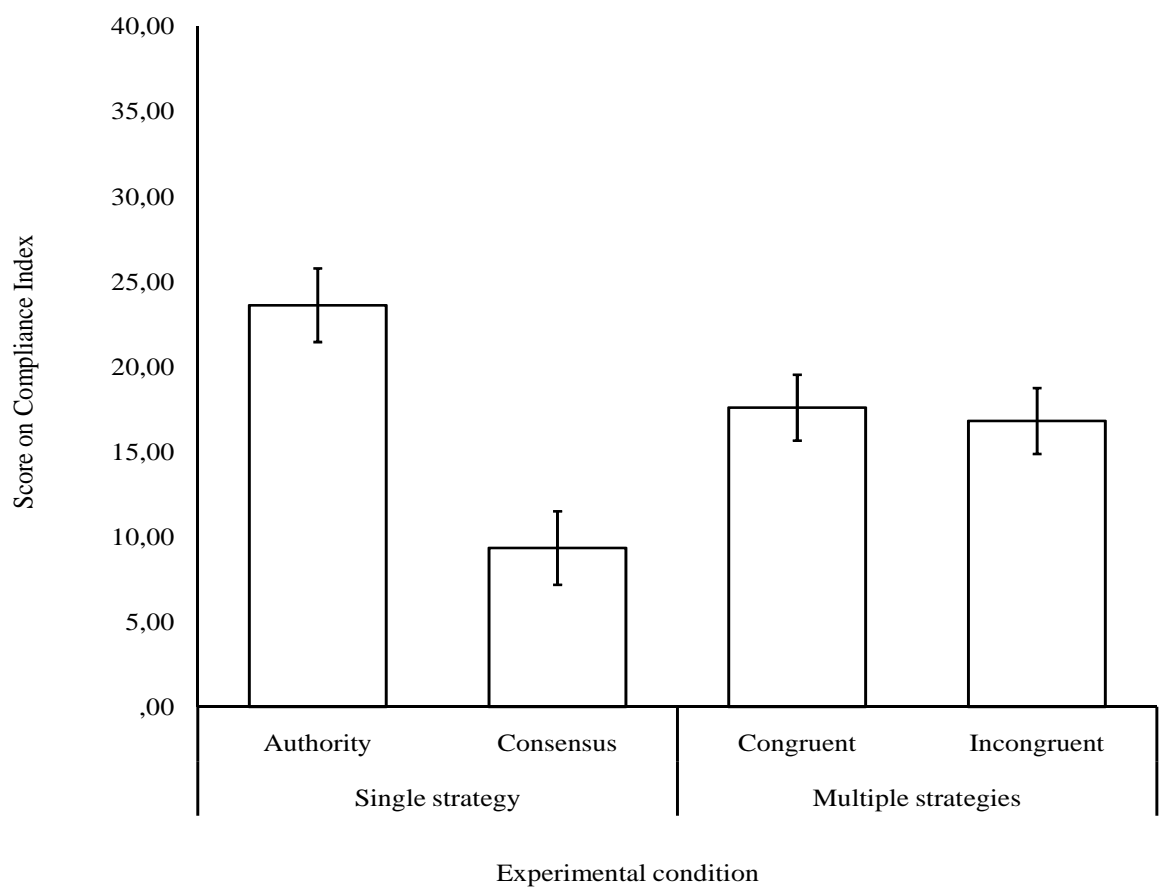

Table 1 Post-hoc comparisons of the four experimental conditions in Study 1

\begin{tabular}{lcccc}
\hline (A) condition & $(B)$ condition & $(\bar{A}-\bar{B})$ & $S E$ & $p$-value \\
\hline MS C & SS Cons & 8.25 & 2.79 & .005 \\
MS C & MS IC & 0.78 & 2.93 & .790 \\
MS C & SS Auth & -6.02 & 2.93 & .046 \\
SS Cons & MS C & -8.25 & 2.79 & .005 \\
SS Cons & MS IC & -7.47 & 2.93 & .015 \\
SS Cons & SS Auth & -14.27 & 2.93 & .001 \\
MS IC & MS C & -0.78 & 2.93 & .790 \\
MS IC & SS Cons & 7.47 & 2.93 & .015 \\
MS IC & SS Auth & -6.8 & 3.06 & .032 \\
SS Auth & MS C & 6.02 & 2.93 & .046 \\
SS Auth & SS Cons & 14.27 & 2.93 & .001 \\
SS Auth & MS IC & 6.8 & 3.06 & .032 \\
\hline
\end{tabular}

Notes: SS - single strategy, MS - multiple strategies, Auth - authority, Cons - consensus, $\mathrm{C}$ - congruent, IC - incongruent. 


\subsubsection{Confidence}

Analysis of the confidence scores (Cronbach's $\alpha=0.783$ ) shows that the average confidence score for the single source conditions, $\bar{X}=7.7, S E=.29$, was similar to that of the multiple strategy condition, $\bar{X}=8.1, S E=.25 t(42)=0.673, p=.504$. Within the single strategy condition, no significant effect of strategy was found: Participants in the authority condition, $\bar{X}=7.8, S E=.52$, were as confident as participants in the consensus condition, $\bar{X}=7.7, S E=.61, t(20)=0.061, p<.952$. Also, different from the previous results on compliance, a significant effect of congruency was found: Within the multiple strategy condition the confidence in the final rating based on advice from incongruent sources, $\bar{X}=7.5, S E=0.17$, was lower than that based on advice from congruent sources, $\bar{X}=8.8, S E=.43, t(20)=2.992$, $p=.007$. Hence, while incongruent advice did not lead to lowered compliance, it did lead to a lowered confidence in the final rankings.

\subsection{Discussion}

Study 1 quantitatively shows human affinity for the preferential strategy, and solely that strategy. Compliance was greatest in the condition where advice came from only the preferential source (expert). Contrary to intuition, having multiple sources of advice agree on the recommendation had not only no positive impact on compliance levels but actually had a slightly negative effect when compared to the preferred strategy (Table 2, italics).

This discrepancy could potentially be a result of increasing cognition and elaboration moving from a strictly peripheral processing approach to a higher elaboration central route. The added advice could have introduced a sense of skepticism or lack of trust with intention of the application (observed in the variance in the usefulness-index across conditions). Additionally, the multiple sources condition could have resulted in lower compliance due to the presence of the lesser preferential consensus source. While weaker than the authority source, as the results show, the consensus source did have a positive effect on compliance when presented individually, however when presented alongside the authority source, the credibility of the stronger (authority) source could have been diminished by the weaker consensus recommendation. Again, this is contrary to common intuition that the persuasive effect of multiple sources is cumulative (i.e., becomes stronger with each additional positive source). Regardless, the results are clear: in some situations, using multiple strategies can be detrimental as compared to presentation of the single preferential strategy.

Equally surprising was the finding that there was no appreciable variation between the multiple strategies condition where the sources agreed and the multiple strategies condition where the sources disagreed. This leads us to believe that individuals mentally default to the preferential strategy and thus any disagreement with the preferential strategy has little effect on final attitudes. These findings are in line with previous research of Tormala and Petty (2004) and Tormala and DeSensi (2009) and demonstrate that conflicting opinions do not affect valence but only attitude certainty (likewise demonstrated by our findings).

It is tempting to assume then that one can simply implement multiple strategies and users will follow the most relevant advice to their cognitive tendencies. While our study showed this to be true, it also showed that the introduction of multiple strategies 
decreases compliance to the end goal and decreased user confidence in the advice provided by the system. It is thus most desirable to implement the single most effective strategy.

\section{Study 2: equal strategies}

Study 1 raised an important question that still needs to be answered to have a clear picture of the correct implementation approach: If there is no clearly preferential strategy (i.e., both sources of advice are equally influential), is a single strategy implementation still optimal? In Study 2, we address this question by conducting Study 1 a second time with equally preferential sources of advice to see if there is any context in which multiple strategies are significantly more effective than a single strategy.

\subsection{Creating equal strategies}

To create implementations of both the consensus and authority strategy that were equally preferred sources of advice in the item-ranking scenario we pre-tested a number of different implementations of both the authority strategy and the consensus strategy in a similar way as we pre-tested participants preference toward the strategy in Study 1: We invited a group of participants via e-mail to choose, after being introduced to the item-ranking task, from which sources they would like advice.

After several changes to our wording and several small pretests, we chose to reword the implementation of the authority strategy to make it less preferential given the item-ranking scenario. Participants were told that they would receive advice originating from a 'doctor'. This was supported with the notion that: "You will get tips on how to better rank your items based on the knowledge of a doctor". The consensus strategy was implemented like Study 1. A pre-test of these two implementations by 69 participants showed that 32 participants wanted to receive advice from the expert, while 37 participants chose the consensus advice. Thus, these implementations in Study 2 enabled us to examine the effect of using a single strategy or multiple strategies when the strategies are equally preferred.

\subsection{Method}

\subsubsection{Participants}

Forty-eight undergraduate students volunteered to participate by accepting an e-mail invitation with a link to the study website. Participants were all from the communication science department of a large west-coast university in the USA. The e-mail invitation was sent to a total of 113 possible participants, giving the study a $42.5 \%$ response rate. The final sample consisted of $28(58.3 \%)$ females. The average age of the sample was $21.3(\mathrm{SD}=2.19)$. None of the participants in Study 2 had previously participated in Study 1. 


\subsubsection{Procedure}

The procedure utilised in Study 2 was identical to that of Study 1, with the exception that the authority source and the consensus source were designed to be equally preferential.

\subsection{Results}

Like in Study 1, for each of the dependent variables we looked at a main-effect of the number of strategies. We then performed separate analysis to test the effects of the specific strategy that was used, the number of strategies used, and the congruence of the messages under the multiple strategy condition.

\subsubsection{Compliance}

For compliance to the advice given in the four experimental conditions, we found no significant main-effect of the number of sources: The average compliance score for the single source conditions, $\bar{X}=19.0, S E=1.49$, was not significantly different from that of the multiple strategy condition, $\bar{X}=16.7, S E=1.70 t(46)=1.029, p=.309$. Within the single strategy condition no effect - as expected based on the pre-test - of the actual strategy that was used was found: Participants in the authority condition, $\bar{X}=19.5, S E=2.34$, complied equally to the advice as participants in the consensus condition, $\bar{X}=18.5, S E=1.97, t(22)=0.300, p=.767$. Within the multiple strategy condition participants in the incongruent group, $\bar{X}=12.3, S E=2.52$, complied less to the advice than those in the congruent group, $\bar{X}=21.1, S E=1.51, t(22)=2.98$, $p<.01$.

Figure 2 shows the means and standard errors for each of the experimental groups. When conducting a one-way four level ANOVA on this data, there is a significant main-effect of condition, $F(3,44)=3.267, p<.05$. Bonferroni corrected pairwise comparisons show that the incongruent condition for the multiple-strategies approach scored significantly lower in compliance than all other conditions (see Table 2). They also show that not only is there no significant difference in compliance between the single authority and single consensus but also no significant difference between either of the single strategies versus the multiple congruent strategies condition. Hence, for equal strength strategies, incongruence in advice does have a negative effect on compliance.

\subsubsection{Confidence}

Analysis of the confidence scores (Cronbach's $\alpha=0.908$ ) shows that the average confidence score for the single source condition, $\bar{X}=7.2, S E=.24$, was similar to that of the multiple strategy condition, $\bar{X}=7.15, S E=.38 t(46)=0.837, p=.407$. Within the single strategy condition, no significant effect of strategy was found: Participants in the authority condition, $\bar{X}=7.3, S E=.36$, were as confident as participants in the consensus condition, $\bar{X}=7.1, S E=.34, t(22)=0.340, p<.737$. No significant effect of congruency was found: Within the multiple strategy condition, the confidence in the final rating based on advice from incongruent sources, $\bar{X}=7.5$, $S E=0.65$, was the same as that based on advice from congruent sources, $\bar{X}=7.5$, 
Combining multiple influence strategies to increase consumer compliance 45

$S E=.41, t(22)=0.001, p=0.99$. These findings show no significant difference in confidence between any of the conditions. This suggests that for equal strength strategies, confidence does not vary despite changes in source quantity and congruence.

Figure 2 The effects of the use of single or multiple strategies, either authority or consensus and congruent or incongruent advice on compliance in Study 2

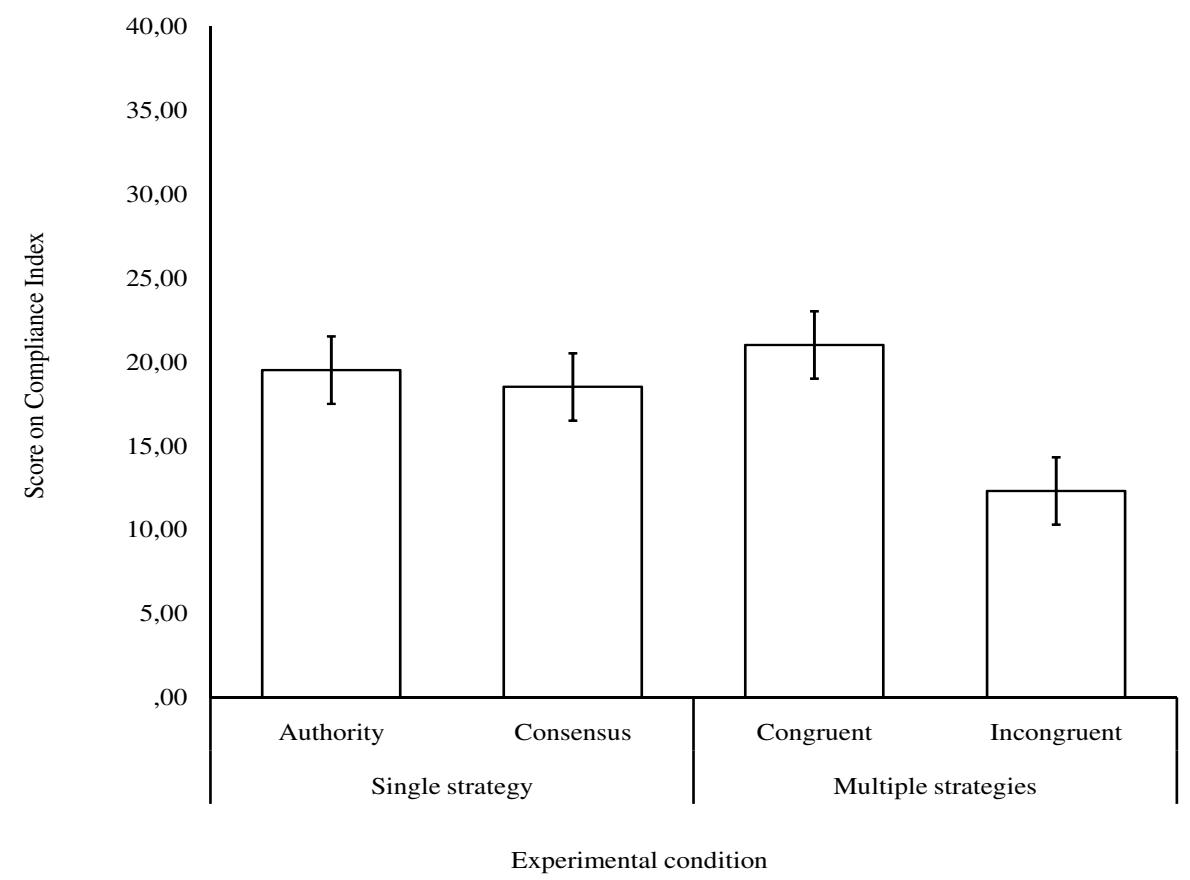

Table 2 Post-hoc comparisons of the four experimental conditions in Study 2

\begin{tabular}{lcccc}
\hline A) condition & $(B)$ condition & $(\bar{A}-\bar{B})$ & $S E$ & $p$-value \\
\hline MS C & SS Cons & 2.5 & 2.99 & .409 \\
MS C & MS IC & 8.8 & 2.99 & .006 \\
MS C & SS Auth & 1.6 & 2.99 & .600 \\
SS Cons & MS C & -2.5 & 2.99 & .409 \\
SS Cons & MS IC & 6.3 & 2.99 & .043 \\
SS Cons & SS Auth & -.92 & 2.99 & .761 \\
MS IC & MS C & -8.8 & 2.99 & .006 \\
MS IC & SS Cons & -6.3 & 2.99 & .043 \\
MS IC & SS Auth & -7.2 & 2.99 & .021 \\
SS Auth & MS C & -1.6 & 2.99 & .600 \\
SS Auth & SS Cons & .92 & 2.99 & .761 \\
SS Auth & MS IC & 7.2 & 2.99 & .021 \\
\hline
\end{tabular}

Notes: SS - single strategy, MS - multiple strategies, Auth - authority, Cons - consensus, $\mathrm{C}$ - congruent, IC - incongruent. 


\subsection{Discussion}

The results from Study 2 demonstrate the importance of designing persuasive strategies with careful mindfulness to the preferentiality of the persuasive source. Additionally, results show that under the condition of equal preference, congruence of persuasive arguments does play an important role in the overall compliance. Finally, the results suggest that confidence may be more heavily influenced but the overall preferability of a source rather than a lack on congruence in persuasive appeals.

As expected, manipulating both the expert and consensus arguments to be equally appealing resulted in both single strategy conditions having equal effects on compliance of the participants. Also, as expected, the confidence scores for participants in both single strategy conditions were not significantly different. From these results it is easy to reason that overall effectiveness of persuasive argument is more contingent on the overall preferability of the argument than the argument type.

More surprising were the effects of equal preferential strategies on the multiple strategies condition. The condition of multiple congruent sources did not demonstrate a significant difference in compliance from either of the single strategy conditions. This shows that even in the case of equally preferable sources, there is no compounding effect on compliance. While no harm was demonstrated in presenting multiple congruent persuasive appeals simultaneously, there was also no benefit. These findings are contrary to the intuition that multiple sources of congruent persuasive attempts strengthen a persuasive appeal in an additive manner.

Further telling was the significant decrease in compliance in the multiple strategies, incongruent condition as these results contradict previous findings. This finding suggests that when two strategies are equally preferential, contradicting arguments can have a significant negative effect on compliance. Given the negative effect on compliance, it is interesting to note that confidence remained unaffected in the incongruent condition having no significant variation from either singles strategy or the multiple congruent strategy. This finding suggests that confidence in advice is primarily based on quality of the sources rather than context of the advice.

\section{Study 3: applied strategic implementations}

Studies 1 and 2 demonstrated the importance of a careful implementation approach for persuasive arguments. They used controlled laboratory manipulations to prove that not only is it the type of persuasive strategy that matters, but more importantly, how and with what other strategies an argument is implemented. The contextual congruence of the implementation was shown to have a significant controlling effect over the potency of the overall persuasive attempt. In Study 3, we take the previous findings and implement the above strategies in a live market setting to demonstrate validity and applicability in a traditional consumer facing advertisement setting. 
Combining multiple influence strategies to increase consumer compliance 47

\subsection{Method}

\subsubsection{Participants}

Two hundred and nineteen thousand five hundred North American Google Search engine users between the ages of 18 and 55 were exposed to one out of six advertisements belonging to one of our experimental conditions.

\subsubsection{Procedure}

Six Google search advertisements were created to solicit users to participate in a study. All six advertisements were titled 'Participate in a study!' with varying 140 character descriptions that fell into two conditions: advertisements using only a single social influence strategy versus those that used multiple social influence strategies. Three social influence strategies were used in this study:
a consensus
b authority
c scarcity.

Our main aim was to compare the performance of an advertisement that implemented all of these strategies to one that implemented only one of these strategies.

In the single strategy condition an implementation of one of the social influence strategies was shown to participants in the textual advertisement to encourage them to participate in our online study:

$1100 \mathrm{~s}$ of others have taken this study before

2 Professor Ford recommends taking this study

3 there are only 18 hours left to participate in this study.

The first strategy implements the influence strategy consensus, the second authority, and the third scarcity.

In the multiple strategy condition, implementations of multiple strategies in a single advertisement were shown to participants. To control for implementation order, we showed one of the following advertisements to participants:

$1100 \mathrm{~s}$ participated, and Professor Ford recommends it. Only 18 hours left.

2 Professor Ford recommends it, 100s participated, only 18 hours left.

3 Only 18 hours left, and Professor Ford recommends it. 100s took it.

The study ran for 21 days and each advertisement was systematically alternated over time to ensure an even distribution over the allocated time period.

Once a participant clicked on the advertisement the success of that advertisement was logged. By computing the number of clicks per the number of views we are able to estimate the effectiveness of the advertisement.

After clicking, participants were taken to a landing page that asked if they would like to participate in a study (informed consent). If they selected 'Yes', they were taken 
to the 'study' page where they were told to rank pictures one at a time based on a scale of one to five of how the picture made them feel. The study consisted of two hundred random pictures shown in sequential order to all participants. The intention was to create an attrition test that few, if any, participants completed as a means of gauging the rigor of compliance for each condition.

\subsection{Measures}

The primary measure of compliance to the conditions was the combined click-through rate (number advertisements clicked on/number of advertisements shown) for the advertisements in each condition. This score measures the direct effectiveness of the multiple versus single strategy conditions. A secondary measure was taken by the number of pictures rated in the attrition test before leaving the study. While the compliance goal of the advertisements was to create a click action as accounted for in the first compliance measure, the attrition score was used as a secondary measure of compliance to quantify any carry-over compliance from the initial implementation.

\subsection{Results}

\subsubsection{Click-through}

Table 3 shows the number of views and clicks on each of the advertisements. Aggregated over the different stimuli in the two conditions, there were 87,356 views and 316 clicks in the single strategy condition. The click-through rate in this condition was $.36 \%$. In the multiple strategy condition the number of views was 109,746 and the number of clicks was 195. This is an average click-through of .18\%. The multiple strategy condition scored significantly lower $C h i^{2}=63.1, p<.001$.

Table 3 Views and click-through rate of the advertisements used in Study 3

\begin{tabular}{|c|c|c|c|c|}
\hline Condition & Add number & Views & Click & Percentage \\
\hline \multirow[t]{3}{*}{ Single } & 1 ' 100 s of others...' & 25,825 & 123 & .47 \\
\hline & 2 'Professor Ford...' & 24,509 & 61 & .25 \\
\hline & 3 'There are only...' & 37,022 & 132 & .36 \\
\hline \multirow[t]{3}{*}{ Multiple } & 1 '100s participated...' & 23,546 & 52 & .22 \\
\hline & 2 'Professor Ford recommends...' & 21,390 & 51 & .24 \\
\hline & 3 'Only 18 hours left...' & 64,810 & 92 & .14 \\
\hline
\end{tabular}

To illustrate the effectiveness of each of the advertisements, we modelled the success of each of the advertisements independently (dotted lines) and aggregated over conditions (solid black and gray lines) as a beta binomial and we plotted the posterior beta densities in Figure 3. It is clear that each of the advertisements that implemented a single strategy scored higher than those implementing multiple strategies. Thus, the effects of the social influence strategies do not seem to add up when used simultaneously in a single advertisement.

These findings are in keeping with the previous laboratory finding from Studies 1 and 2 . The results continue to fail to show any benefit to combining multiple strategies 
Combining multiple influence strategies to increase consumer compliance 49

into single persuasive appeal while suggesting single strategies are the most effective persuasive technique.

Figure 3 Model-based click-through behaviour based on the different advertisement versions

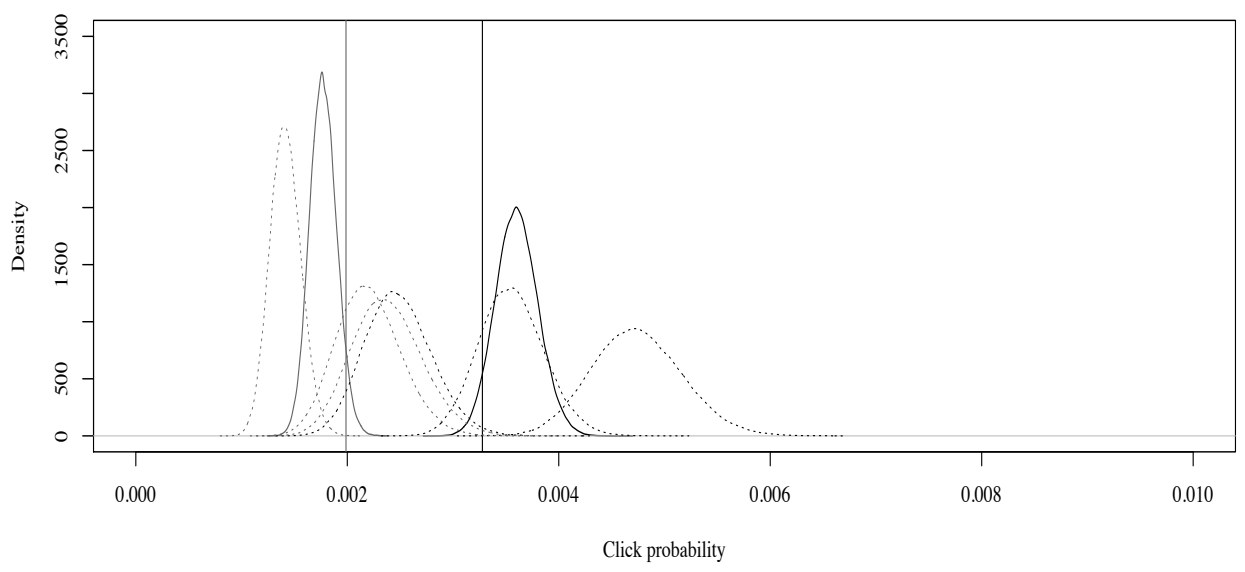

Note: The black line represents the average click through for the multiple strategies advertisements, while the tray line represents the average click through for the single strategy advertisements.

\subsubsection{Attrition}

To see whether the differences in persuasion are only present when clicking on the advertisements or whether they also lead to a more active participation in the final cause - in this study the attrition test - we compare the number of pictures rated by participants that clicked on an advertisement implementing multiple strategies versus those that clicked on an advertisement implementing a single strategy.

Of the 316 participants that clicked on an image with a single strategy, 208 rated at least one image. In this condition a total of 3,528 images were rated and thus on average participants rated 16.9 images. Of the 195 participants that arrived to the attrition task by clicking on a advertisement that implemented multiple strategies, 106 actually rated at least one picture. In this condition a total of 1,893 images were rated and thus participants rated on average 17.9 images. This difference in attrition between the two conditions is not statistically significant.

These finding suggest a finite temporal nature to the persuasive effectiveness of an argument. The single strategy was more effective at convincing participants to take action on the study by both clicking on the initial advertisement and by clicking through the waver to begin the study. However, the actual attrition test proved equally wearing on both groups as there was no significant difference in depth of participation between those in the single strategy group versus the multiple strategy group. 


\subsection{Discussion}

Study 3 confirmed the applicability of Studies 1 and 2 in a live market setting. By providing further evidence that a single strategy is more effective than multiple strategies, the results demonstrate the importance of tactfully selecting a persuasive strategy implementation approach.

While as a whole, the single strategy was more effective, it is important to note that the authority argument fared poorly compared to the consensus and scarcity arguments. The authority argument was clearly least preferential and was even near on par with some of the multiple strategy approaches. This result, combined with our findings in the previous studies, demonstrate the individuals do not disregard non-preferential strategies when sequenced with other preferential strategies. Even when two preferential strategies are combined with a less preferential strategy, the results of sequential usage of multiple persuasive strategies never leads to a beneficial outcome and can, as shown here, lead to detrimental effects.

\section{General conclusions}

This paper used three studies to carefully explore the difference in persuasiveness between single strategy and multi strategy persuasive appeals. The results failed to demonstrate any positive effects to combining multiple persuasive strategies into one persuasive appeal. In fact, the results consistently demonstrated a detrimental effect of implementing multiple strategies simultaneously.

Study 1 demonstrated the significance of a preferential strategy and showed, for the first time, a potentially negative affect of multiple strategy implementation. In Study 2, we carefully controlled for preference of persuasive strategy by exhaustively pre-testing strategies to remove all significant bias in preferentiality. The resulting findings demonstrated that even when two preferential strategies are combined into a single appeal, the results do not outweigh either of the individual strategies. These findings have significant implication for industrial practice as they clearly show the lack of reward for carefully devising equally preferential strategies to implement simultaneously.

Finally, in Study 3 we took our laboratory findings and devised a live, 343,000 impression advertisement manipulation to test our results in a directly applicable setting. The results confirmed our laboratory findings and soundly affirmed our conclusions that the sequential implementation of multiple persuasive strategies simultaneously does not have a net positive effect on compliance, as was the case in Study 3, but has a negative effect on compliance.

\subsection{Persuasion in interactive advertising}

The results presented in this paper have clear implications for the practices of interactive marketing and advertising. While marketers are already concerned with optimising the product offers or propositions that are made to individual customers, by and large the persuasive strategies or influence tactics that are used are added to the appeal in a rather ad-hoc manner. Interactive marketers, while more and more frequently resorting to the use of influence tactics often use multiple tactics to support a similar proposition. Our 
results, however, show that this does not maximise the effect of the use of influence tactics in interactive marketing: a careful selection of a specific strategy that fits the appeal, or possibly the individual consumer, leads to higher compliance and thus in the end to higher revenues.

In practice, interactive advertisers and marketers already have to limit their selection of influence tactics that are used in their campaigns because of space limitations of the medium or time constraints of consumers. We, however, introduce an additional motivation to carefully test, and eventually select individual strategies. The use of multiple strategies can confuse the arguments made to consumers, resulting in higher elaboration, less peripheral processing, and eventually a smaller effect of the tactics that are used. As a result of these findings, we would caution practitioners against using multiple persuasive appeals simultaneously whenever possible. Rather, we would suggest finding a single preferential persuasive appeal for given context and implement only that appeal in order to produce maximum compliance in the end user.

\subsection{Future research}

This research highlighted the importance of selection appropriate influence tactics. However, the results that we have presented are based on the behaviour of groups of people. The next step in this research is to see whether there are differences between individuals in their responses to influence tactics. In the psychology literature, such stable individual differences have already been described (e.g., Kardes et al., 2007; Nail et al., 2001) and theorists in human-computer interaction (Kaptein et al., 2011b) and information systems (Kaptein, 2011) have described how adaptation to such individual differences can increase compliance to persuasive appeals. This focus on individual differences in susceptibility to different types of propositions - rather than to the actual end goal of the proposition - is in-line with recent developments in online marketing (see e.g., Hauser et al., 2009). The results presented in this article should be evaluated with respect to individual level responses to influence tactics so that the single 'right' tactic can be selected not just at an average level, but, ultimately, also at the level of individual consumers.

\section{Acknowledgements}

The authors would like to thank Estella Maria Go for her help in collecting the data in Studies 1 and 2. We would also like to thank Professor Clifford Nass, Professor Panos Markopoulos, Professor Emile Aarts, and Dr. Boris de Ruyter for their insightful comments on earlier versions of this manuscript.

\section{References}

Ajzen, I. and Fishbein, M. (1980) Understanding Attitudes and Predicting Social Behavior, Prentice-Hall, Englewood Cliffs, NJ.

Barry, B. and Shapiro, D.L. (1992) 'Influence tactics in combination: the interactive effects of soft versus hard tactics and rational exchange', Journal of Applied Social Psychology, Vol. 22, No. 18, pp.1429-1441. 
Blass, T. (1991) 'Understanding behavior in the Milgram obedience experiment: the role of personality, situations, and their interactions', Journal of Personality and Social Psychology, Vol. 60, No. 3, pp.398-413.

Booth-Butterfield, S. and Welbourne, J. (2002) 'The elaboration likelihood model: its impact on persuasion theory and research', in Dillard, J.P. and Pfau, M.W. (Eds.): The Persuasion Handbook Developments in Theory and Practice, pp.195-211, Sage.

Brock, T.C. (1968) 'Implications of commodity theory for value change', in Greenwald, A.G., Brock, T.C. and Ostrom, T.M. (Eds.): Psychological Foundations of Attitudes, pp.243-275, Academic Press, New York.

Cialdini, R. (2001) Influence, Science and Practice, Allyn \& Bacon, Boston.

Cialdini, R. (2004) 'The science of persuasion', Scientific American Mind, Vol. 14, pp.70-78.

Cialdini, R.B. (2005) 'Don't throw in the towel: use social influence research', American Psychological Society Observer, Vol. 18, No. 4, pp.33-34.

Eisend, M. (2008) 'Explaining the impact of scarcity appeals in advertising: the mediating role of perceptions of susceptibility', Journal of Advertising, Vol. 37, No. 3, pp.33-40.

Falbe, C.M. and Yukl, G. (2008) 'Consequences for managers of using single influence tactics and combinations of tactics', The Academy of Management Journal, Vol. 35, No. 3, pp.638-652.

Fromkin, H.L. (1970) 'Effects of experimentally aroused feelings of undistinctiveness upon valuation of scarce and novel experiences', Journal of Personality and Social Psychology, Vol. 16, No. 3, pp.521-529.

Goldstein, N.J., Cialdini, R.B. and Griskevicius, V. (2008) 'A room with a viewpoint: using social norms to motivate environmental conservation in hotels', Journal of Consumer Research, Vol. 35, No. 3, pp.472-482.

Hardin, C.D. and Higgins, E.T. (1996) 'Shared reality: how social verification makes the subjective objective', in Sorrentino, Richard M., and Higgins, E. Tory (Eds.): Handbook of Motivation and Cognition, Vol. 3: The Interpersonal Context. Handbook of Motivation and Cognition, pp.28-84, Guilford Press, New York, NY, USA.

Hauser, J.R., Urban, G.L., Liberali, G. and Braun, M. (2009) 'Website morphing', Marketing Science, Vol. 28, No. 2, pp.202-223.

Inman, J., Peter, A. and Raghubir, P. (1997) 'Framing the deal: the role of restrictions in accentuating deal value', Journal of Consumer Research, Vol. 24, No. 1, pp.68-79.

Kaptein, M.C. and Eckles, D. (2010) 'Selecting effective means to any end: futures and ethics of persuasion profiling', in Ploug, T., Hasle, P. and Oinas-Kukkonen, H. (Eds.): Persuasive Technology, pp.82-93, Springer Berlin/Heidelberg.

Kaptein, M.C., Duplinsky, S., and Markopoulos, P. (2011a) 'Means based adaptive persuasive systems. In Proceedings of the 2011 Annual Conference on Human Factors in Computing Systems, CHI '11, pp.335-344, ACM, New York, NY, USA.

Kaptein, M.C., Eckles, D. and Davis, J. (2011b) 'Envisioning persuasion profiles: challenges for public policy and ethical practice', ACM Interactions, Vol. 18, No. 5, pp.66-69.

Kaptein, M.C. (2011) 'Adaptive persuasive messages in an e-commerce setting: the use of persuasion profiles', in Proceedings of ECIS 2011, Helsinki.

Kardes, F.R., Fennis, B.M., Hirt, E.R., Tormala, Z.L. and Bullington, B. (2007) 'The role of the need for cognitive closure in the effectiveness of the disrupt-then-reframe influence technique', Journal of Consumer Research, Vol. 34, No. 3, pp.377-385.

Kelman, C. and Hamilton, V.L. (1989) Crimes of Obedience: Toward a Social Psychology of Authority and Responsibility, Yale University Press, New Haven.

Lynn, M. (1989) 'Scarcity effects on desirability: mediated by assumed expensiveness?', Journal of Economic Psychology, Vol. 10, No. 2, pp.257-274. 
Combining multiple influence strategies to increase consumer compliance 53

Lynn, M. (1991) 'Scarcity effects on value: a quantitative review of the commodity theory literature', Psychology and Marketing, Vol. 8, No. 1, pp.43-57.

Martin, R. and Hewstone, M. (2003) 'Social influence processes of control and change: conformity, obedience to authority, and innovation', in Hogg, M.A. and Cooper, J. (Eds.): Psychological Foundations of Attitudes, pp.347-366, Sage, London.

Milgram, S. (1974) Obedience to Authority, Tavistock, London.

Modigliani, A. and Rochat, F. (1995) 'The role of interaction sequences and the timing of resistance in shaping obedience and defiance to authority', Journal of Social Issues, Vol. 51, No. 3, pp.107-123.

Nail, P.R., Correll, J.S., Drake, C.E., Glenn, S.B., Scott, G.M. and Stuckey, C. (2001) 'A validation study of the preference for consistency scale', Personality and Individual Differences, Vol. 31, No. 7, pp.1193-1202.

Petty, R.E. and Cacioppo, J.T. (1986) 'The elaboration likelihood model of persuasion', Advances in Experimental Social Psychology, Vol. 19, No. 1, pp.123-205.

Petty, R.E. and Wegener, D.T. (1999) 'The elaboration likelihood model: current status and controversies', in Chaiken, S. and Trope, Y. (Eds.): Dual-process Theories in Social Psychology, pp.41-72, Guilford Press, New York.

Rhoads, K. (2007) 'How many influence, persuasion, compliance tactics \& strategies are there?' [online] http://www.workingpsychology.com/numbertactics.htm (accessed 12-10-2011).

Snyder, C.R. and Fromkin, H.L. (1980) Uniqueness: The Human Pursuit of Difference, Springer-Verlag, New York.

Tormala, Z.L. and DeSensi, V.L. (2009) 'The effects of minority/majority source status on attitude certainty: a matching perspective', Personality and Social Psychology Bulletin, Vol. 35, No. 1, pp.114-125.

Tormala, Z.L. and Petty, R.E. (2004) Resistance to Persuasion and Attitude Certainty: The Moderating Role of Elaboration, Technical Report 11, Department of Psychology, Indiana University, Bloomington, IN, USA.

West, S.G. (1975) 'Increaing the attractiveness of college cafeteria food: a reactance theory perspective', Journal of Applied Psychology, Vol. 60, No. 5, pp.656-658.

Zhu, F. and Zhang, X.M. (2010) 'Impact of online consumer reviews on sales: the moderating role of product and consumer characteristics', Journal of Marketing, Vol. 74, No. 2, pp.133-148. 